\title{
The relationship between neutrophil-to-lymphocyte ratio and albuminuria in type 2 diabetic patients: a pilot study
}

\author{
Cüneyt Kahraman ${ }^{1}$, Nilüfer Kuzeyli Kahraman², Bekir Aras $^{3}$, Süleyman Coşgun ${ }^{1}$, Erim Gülcan ${ }^{1}$
}

\author{
${ }^{1}$ Department of Internal Medicine, Dumlupinar University, School of Medicine, \\ Kutahya, Turkey \\ 2Department of Internal Medicine, DPU Evliya Çelebi Research and Education \\ Hospital, Kutahya, Turkey \\ ${ }^{3}$ Department of Urology, Dumlupinar University, School of Medicine, Kutahya, Turkey
}

Submitted: 29 August 2014

Accepted: 3 November 2014

Arch Med Sci 2016; 12, 3: 571-575

DOI: $10.5114 /$ aoms.2016.59931

Copyright $\odot 2016$ Termedia \& Banach

\author{
Corresponding author: \\ Cüneyt Kahraman MD \\ Dumlupinar University \\ School of Medicine \\ Evliya Celebi \\ Hastanesi Ana Bina 1. \\ Kat Dahiliye Poliklinigi \\ 43100 Kutahya, Turkey \\ Phone: +905057454399 \\ E-mail: kahramandoktor@ \\ gmail.com
}

\begin{abstract}
Introduction: Diabetes mellitus (DM) has become a global economic burden due to treatment costs and attendant complications. Albuminuria is the precursor of end stage renal failure and is an inflammatory process. In the recent past, it has been reported that the neutrophil/lymphocyte ratio (NLR), which is a cost-effective and accessible marker, may be a favorable indicator of the inflammatory status. The aim of this study was to investigate the relationship between the neutrophil/lymphocyte ratio and the presence and level of diabetic nephropathy (DN).

Material and methods: A total of 112 patients with type-2 DM who were followed by our internal medicine and nephrology clinics between February 2013 and June 2014 were included in this pilot study and were retrospectively evaluated. All participants had a 24-hour urinary albumin excretion (UAE) record. Demographic parameters, biochemical parameters and albuminuria levels were recorded. Patients were divided into three groups according to their level of albuminuria.

Results: Significant differences were detected between the groups in terms of NLR $(p<0.001)$. There was a linear increase in NLR in parallel to the increase in 24 -hour UAE mean values $(p<0.001)$. A positive correlation was detected between NLR and C-reactive protein, urea, creatinine, and red cell distribution width. However, 24-hour UAE was negatively correlated with lymphocyte count $(p<0.001)$.

Conclusions: A high degree of correlation was determined among albuminuria, glomerular filtration rate and NLR levels. These results may suggest the notion that diabetic nephropathy involves an inflammatory process.
\end{abstract}

Key words: albuminuria, diabetic nephropathy, inflammation, neutrophil/ lymphocyte ratio.

\section{Introduction}

Type 2 diabetes mellitus (DM) can have serious socio-economic effects due to its many potential complications, which include microvascular (diabetic nephropathy (DN), neuropathy and retinopathy) and macrovascular complications (atherosclerosis, ischemic heart disease, stroke and periph- 
eral vascular disease, which frequently results in amputation) [1]. Diabetic nephropathy (DN) leads to significant problems in $25-40 \%$ of diabetic patients and is the major cause of end stage renal failure [2]. The urine microalbumin excretion rate (UMAER) can be used to detect and monitor the progression of DN [3].

Inflammation is triggered in case of increased cardiovascular disease risk such as insulin resistance, visceral obesity, metabolic syndrome and type 2 DM [4]. Thus, elevated systemic inflammatory markers have been associated with a high prevalence of cardiovascular diseases [5, 6]. Some cardiovascular risk factors including hypertension, DM, hyperlipidemia, obesity and smoking are associated with chronic low-grade inflammation [7]. Inflammation plays a major role in development and progression of DN, and many inflammatory cytokines (interleukin-1 (IL-1), interleukin-6 (IL-6), interleukin-8 (IL-18), tumor necrosis factor $\alpha$ (TNF- $\alpha$ ), etc) are closely related to the pathogenesis of $\mathrm{DN}$ [8]. The $\mathrm{CD}_{4} / \mathrm{CD}_{8}$ ratio decreases in the case of DM. Circulating leukocyte rates are changed during the inflammatory response. Neutrophilia is accompanied by relative lympho- penia $[9,10]$. An index has recently been generated to reflect both neutrophil elevation, which demonstrates the acute state of inflammation, and lymphopenia, which occurs following physiological stress. This index, i.e. the neutrophil/lymphocyte ratio, has been suggested as a favorable indicator of the inflammatory status [11, 12].

\section{Material and methods}

A total of 112 patients with type-2 DM who were followed by our internal medicine and nephrology clinics between February 2013 and June 2014 were included in this pilot study and were retrospectively evaluated. Patients were divided into three subgroups as follows: normoalbuminuria (38 females, 16 males; 48.2\%), microalbuminuria (18 females, 16 males; 30.4\%) and macroalbuminuria (17 females, 7 males; $21.4 \%$ ). Patient characteristics are given in Table I. All participants had a 24-hour urinary albumin excretion (UAE) record. Age, gender and DM duration were recorded as well as several biochemical and hematological parameters (hemoglobin, white blood cell (WBC), platelet count, mean platelet volume (MPV), neutrophil

Table I. Demographic and laboratory parameters of the patients

\begin{tabular}{|c|c|c|c|c|}
\hline Variables & $\begin{array}{l}\text { Normoalbuminuria } \\
\qquad(N=54)\end{array}$ & $\begin{array}{l}\text { Microalbuminuria } \\
\qquad(N=34)\end{array}$ & $\begin{array}{c}\text { Macroalbuminuria } \\
\qquad(N=24)\end{array}$ & $P$-value \\
\hline Age [years] & $59.9 \pm 7.9(42-83)$ & $64.1 \pm 8.9(33-84)$ & $56.8 \pm 12.5(30-74)$ & 0.013 \\
\hline Gender (\% female) & 70.3 & 52.9 & 70.8 & 0.200 \\
\hline Duration of diabetes [years] & $5.0 \pm 3.2(1-19)$ & $5.6 \pm 4.0(1-15)$ & $5.4 \pm 3.3(1-14)$ & 0.742 \\
\hline $\mathrm{HbA}_{1 \mathrm{c}}(\%)$ & $9.9 \pm 2.4(5.5-14.1)$ & $8.6 \pm 2.5(5.4-15.2)$ & $8.4 \pm 2.5(5.2-13.6)$ & 0.044 \\
\hline Total cholesterol [mg/dl] & $202.4 \pm 55.5(74-358)$ & $198.0 \pm 50.0(92-342)$ & $182.7 \pm 43.4(126-277)$ & 0.300 \\
\hline Triglyceride [mg/dl] & $193.7 \pm 146.5(49-988)$ & $210.6 \pm 129.0(65-593)$ & $171.6 \pm 96.8(43-476)$ & 0.552 \\
\hline HDL-cholesterol [mg/dl] & $47.5 \pm 11.0(24-70)$ & $40.6 \pm 12.4(5-71)$ & $51.0 \pm 15.9(26-108)$ & 0.008 \\
\hline LDL cholesterol [mg/dl] & $119.5 \pm 42.7(26-270)$ & $120.0 \pm 40.7(45-258)$ & $103.2 \pm 42.5(57-225)$ & 0.243 \\
\hline Serum urea $[\mathrm{mg} / \mathrm{dl}]$ & $34.9 \pm 12.4(18-74)$ & $43.3 \pm 25.0(18-139)$ & $44.6 \pm 19.2(21-93)$ & 0.053 \\
\hline Creatinine $[\mathrm{mg} / \mathrm{dl}]$ & $0.8 \pm 0.2(0.43-1.46)$ & $1.0 \pm 0.3(0.45-1.88)$ & $1.2 \pm 0.6(0.53-2.51)$ & $<0.001$ \\
\hline $\mathrm{GFR}[\mathrm{ml} / \mathrm{min}]$ & $121.1 \pm 84.6(25-456)$ & $118.0 \pm 90.9(8-441)$ & $85.1 \pm 61.1(22-221)$ & 0.200 \\
\hline Albumin [g/dl] & $4.1 \pm 0.7(2-4.9)$ & $4.1 \pm 0.6(2.3-5.5)$ & $3.9 \pm 0.5(2.7-4.6)$ & 0.570 \\
\hline $\mathrm{CRP}[\mathrm{mg} / \mathrm{l}]$ & $1.6 \pm 3.6(0.1-18.2)$ & $1.4 \pm 1.8(0.1-6.4)$ & $1.7 \pm 1.7(0.1-4.9)$ & 0.970 \\
\hline WBC $\left[\times 10^{3} / \mathrm{mm}^{3}\right]$ & $7.5 \pm 1.9(4.5-14.9)$ & $7.5 \pm 2.2(3.9-12.9)$ & $7.8 \pm 2.1(4.6-14.9)$ & 0.846 \\
\hline Hemoglobin level [g/dl] & $13.5 \pm 1.7(9.4-17.6)$ & $13.4 \pm 1.4(10.5-18.5)$ & $12.2 \pm 1.7(9.3-15.8)$ & 0.006 \\
\hline Platelet count $\left[\times 10^{3} / \mathrm{mm}\right]$ & $259.0 \pm 77.5(101-495)$ & $222.9 \pm 68.5(54-376)$ & $257.0 \pm 100.0(122-588)$ & 0.104 \\
\hline MPV [fl] & $8.8 \pm 1.0(6.6-12.9)$ & $8.8 \pm 1.2(6.9-11.6)$ & $8.3 \pm 0.9(6.9-10.4)$ & 0.174 \\
\hline Neutrophil count $\left[\times 10^{9} / 1\right]$ & $4.3 \pm 1.5(2.1-9.8)$ & $4.8 \pm 1.7(2.2-8.3)$ & $5.4 \pm 1.4(3.1-8.4)$ & 0.022 \\
\hline Lymphocyte count $\left[\times 10^{9} / 1\right]$ & $2.5 \pm 0.8(0.9-4.1)$ & $1.9 \pm 0.7(0.7-3.7)$ & $1.6 \pm 0.6(0.5-4.0)$ & $<0.001$ \\
\hline NLR & $1.9 \pm 0.9(0.73-6.23)$ & $2.6 \pm 1.0(1.09-5.36)$ & $3.6 \pm 1.3(2.1-9.2)$ & $<0.001$ \\
\hline RDW & $14.3 \pm 1.7(12.1-18.8)$ & $14.0 \pm 1.7(12.4-20.9)$ & $15.3 \pm 1.7(11.8-19.1)$ & 0.014 \\
\hline 24-h UAE & $18.0 \pm 14.9(2-28)$ & $105.7 \pm 69.0(33-285)$ & $665.5 \pm 391.9(320-1643)$ & $<0.001$ \\
\hline
\end{tabular}


count, lymphocyte count, neutrophil/lymphocyte ratio (NLR), red cell distribution width (RDW), urea, creatinine, total cholesterol, high-density lipoprotein (HDL) cholesterol, low-density lipoprotein (LDL) cholesterol, triglyceride (TG), hemoglobin $A_{1 c}\left(H b A_{1 c}\right)$, albumin), C-reactive protein (CRP), 24-hour protein and albumin levels. Glomerular filtration rate (GFR) was calculated according to the Modification of Diet in Renal Disease (MDRD) formula. Patients were divided into 3 groups, according to their level of albuminuria, designated as normoalbuminuria (0-30 mg/day), microalbuminuria (30-300 mg/day) and macroalbuminuria (> $300 \mathrm{mg} /$ day). The study excluded patients with active urinary infection, leukocytosis, malignancy, nephrotic syndrome and those who were on steroid use for any reason.

\section{Statistical analysis}

Statistical analysis was done by SPSS version 19.0 (SPSS, Chicago, IL). Data were given as mean \pm standard deviation. Group comparisons were performed via one-way ANOVA and LSD post-hoc tests and relationships between measurements were calculated via Spearman's correlation test. Linear regression was performed to determine the independent factors affecting UAE. P-values less than 0.05 were considered as statistically significant.

\section{Results}

No significant difference was observed between the groups with regard to gender, DM duration, total cholesterol, triglyceride, LDL, GFR, albumin, CRP, total WBC, platelet count and MPV. However, there were significant differences among the 3 groups with regard to age $(p=0.013), \mathrm{HbA}_{1 c}$ $(p=0.044), \mathrm{HDL}(p=0.008)$, creatinine $(p<0.001)$, hemoglobin level $(p=0.006)$, neutrophil count ( $p=0.022)$, lymphocyte count $(p<0.001)$, NLR $(p<0.001)$, RDW $(p=0.014)$ and 24-hour UAE $(p=0.001)$ (Table I).

Neutrophil/lymphocyte ratio did not differ between genders. Neutrophil/lymphocyte ratio correlated with GFR $(r=-0.298, p=0.002)$, hemoglobin $(r=-0.244, p=0.010)$, hematocrit $(r=-0.230$, $p=0.015)$, neutrophil count $(r=0.500, p<0.001)$, lymphocyte count $(r=-0.634, p<0.001)$, CRP $(r=0.309, p=0.033)$, urea $(r=271, p=0.005)$, creatinine $(r=0.304, p=0.001)$, spot urine microalbumin $(r=0.396, p<0.001)$, RDW $(r=0.269$, $p=0.004)$ and total cholesterol $(r=-0.214$, $p=0.025)$. Furthermore, 24-hour UAE correlated with neutrophil count $(r=0.274, p=0.003)$, lymphocyte count $(r=-0.340, p<0.001)$, NLR $(r=0.432, p<0.001)$, hemoglobin $(r=-0.265$, $p=0.005)$, hematocrit $(r=-0.247, p=0.009)$, and creatinine $(r=0.262, p=0.005)$. Glomerular filtration rate correlated with lymphocyte count $(r=0.307, p=0.001)$ and CRP $(r=-0.305, p=0.040)$.

Neutrophil/lymphocyte ratio significantly increased in parallel to albuminuria levels: $1.9 \pm 0.9$, $2.6 \pm 1.0$ and $3.6 \pm 1.3$ for normo-, micro- and macroalbuminuria groups respectively $(p=0.003$ for normo-vs. microalbuminuria groups, $p=0.001$ for micro- vs. macroalbuminuria groups and $p<0.001$ for normo- vs. macroalbuminuria). Neutrophil counts were significantly different only between the normoalbuminuria and macroalbuminuria groups $(p=0.006)$, whereas lymphocyte counts differed significantly between the normoalbuminuria and microalbuminuria $(p=0.002)$ and between the normoalbuminuria and macroalbuminuria groups $(p<0.001)$. In linear regression analysis to reveal the independent factors for UAE, we found that NLR ( $p=0.043, r=0.309), \mathrm{WBC}(p=0.046$, $r=0.302)$ and hemoglobin $(p=0.020, r=-0.366)$ were independent parameters.

\section{Discussion}

While glomerular damage is considered as an early sign of DN, microalbuminuria is a strong indicator of DN progression [13, 14]. Certain authors have suggested that increased protein filtration and reabsorption are related to tubulointerstitial disease and progressive loss of renal function [15]. Diabetic nephropathy-related increase in proteinuria is a part of the cascade of clinical events involving increased blood pressure and progressive decrease in GFR. Glomerular damage gives rise to proteinuria and progressive renal damage in DM. Consequently, fibrosis and inflammation of the collective tubules result in progressive loss of functional nephrons $[16,17]$. Furthermore, DM is not only a metabolic disorder. It is now recognized that several molecules associated with inflammation play a major role in development of DM and DM-related complications [18, 19]. Experimental and clinical studies have demonstrated the significant role of inflammatory molecules (including adipokines, chemokines, adhesion molecules and cytokines) and endothelial dysfunction in the setting of DN [20]. Moreover, it has also been reported that renal inflammation has a crucial place in development and progression of DN [8]. In a study conducted by Spranger et al., it was suggested that circulating inflammatory cytokines modify the development of type 2 DM; elevation of IL- 6 and IL-1 together increased the risk of type 2 DM [21].

White blood cell count and its subtypes, neutrophil count and NLR represent the balance between neutrophil and lymphocyte levels in the body and can be indicators of systemic inflammation. Neutrophil/lymphocyte ratio can be easily calculated as the neutrophil-to-lymphocyte ratio in peripher- 
al blood [22, 23]. There are a limited number of studies investigating the relationship between WBC count and DN. Agarwal and Light found a significant association between WBC count and the prevalence of DN [24]. Furthermore, these authors also emphasized that a positive correlation was found between WBC, neutrophil count and albuminuria level, whereas there was a negative correlation between WBC and lymphocyte count, which in turn may suggest that albuminuria level is related to the inflammatory response [24]. Afşar reported that an increased N/L ratio was independently related to both 24-hour urinary protein and urinary albumin excretion [25]. Abdul-Rahman et al. reported that UAE and hemoglobin levels did not correlate at all [26]. Watts et al. mentioned that glycated hemoglobin $(\mathrm{HbA})$ is an independent factor in increased albuminuria [27]. Also, Akbaş et al. reported that $\mathrm{HbA}_{1 \mathrm{c}}$ and NLR ratio are independent predictors for albuminuria [28]. Similarly, when we performed a linear analysis on our established data, we found that NLR, WBC and hemoglobin were independent factors for albuminuria. Data obtained in our study correspond to the data of this study. Sela et al. compared the blood polymorphonuclear leukocyte (PMNL) counts among 3 patient groups (i.e. CKD, renal replacement therapy (RRT) and continuous ambulatory peritoneal dialysis (CAPD) groups) and a healthy control group [29]. They reported that PMNL counts were significantly higher in these three groups, when compared with the control group; a negative correlation was also observed between PMNL count and GFR. Our study yielded a negative correlation between GFR and NLR levels, whereas there was a positive correlation between NLR and CRP and between GFR and lymphocyte count.

Okyay et al. reported that NLR had a positive correlation with IL-6 and was highly sensitive to C-reactive protein (hs-CRP) [30]. Similarly, our study revealed a negative correlation between NLR and hemoglobin, hematocrit and total cholesterol levels and a positive correlation with CRP. These results suggest that NLR measurements may be used as a marker of the systemic inflammatory response in CKD. Our results also showed that NLR can be used together with albuminuria to detect DN during the follow-up of type 2 diabetic patients. In a study by Akbaş et al., it was found that the neutrophil to lymphocyte ratio was higher in the diabetic patient group, whereas there was no significant difference in total lymphocyte count [28]. Latter, these authors also reported that there was a significant difference among the micro-, normo- and macro-albuminuria groups [31]. In another study, Huang et al. found a significant difference between the diabetic patient group and controls by means of neutrophil and lymphocyte counts [32]. In our study, we found that neutro- phil and lymphocyte counts were different among the groups, which is in accordance with the previous studies. Also, $\mathrm{HbA}_{1 \mathrm{c}}$, total cholesterol and LDL levels were lower in the normoalbuminuria group than in the other groups. This may due to the most frequent follow-up visits of the micro- and macro-albuminuric patients to firmly control the serum cholesterol and $\mathrm{HbA}_{1 \mathrm{c}}$ levels along with the drug use status.

The current study also has some limitations. First of all, we had to study a relatively small number of patients $(n=112)$, as the study was designed in a retrospective manner. Additionally, we could not access and record any blood pressure data of our participant patients, which could in turn suggest that there may have been a negative effect of hypertension on neutrophil and lymphocyte ratio. Moreover, there was some missing information on drugs used by the patients. We were not able to find out which drugs had been used by some of our participants.

In conclusion, in the present study, the possible associations between albuminuria levels, GFR and NLR were investigated in diabetic patients. Neutrophil/lymphocyte ratio was significantly increased in parallel to albuminuria levels and it was negatively correlated with GFR and positively with CRP. These results support the fact that DN involves an inflammatory process and that NLR may represent a marker of this inflammation. Therefore, NLR may serve as a cost-effective and readily accessible marker of DN. The clinical utility of NLR in the case of DN remains to be established in future studies.

\section{Conflict of interest}

The authors declare no conflict of interest.

\section{References}

1. Wild S, Roglic G, Green A, et al. Global prevalence of diabetes: estimates for the year 2000 and projections for 2030. Diabetes Care 2004; 27: 1047-53.

2. Ritz E, Rychlik I, Locatelli F, Halimi S. End-stage renal failure in type 2 diabetes: a medical catastrophe of worldwide dimensions. Am J Kidney Dis 1999; 34: 795-808.

3. Wang T, Wang Q, Wang Z, et al. Diagnostic value of the combined measurement of serum Hcy, serum Cys $\mathrm{C}$, and urinary microalbumin in type 2 diabetes mellitus with early complicating diabetic nephropathy. ISRN Endocrinol 2013; 2013: 407452

4. Sibel S, Cicero A. Impact of physical activity on inflammation: effects on cardiovascular disease risk and other inflammatory conditions. Arch Med Sci 2012; 8: 794-804.

5. Folsom AR, Rosamond WD, Shahar E, et al. Prospective study of markers of hemostatic function with risk of ischemic stroke. The Atherosclerosis Risk in Communities (ARIC) Study Investigators. Circulation 1999; 100: 736-42.

6. Tondi P, Santoliquido A, Di Giorgio A, et al. Endothelial dysfunction as assessed by flow-mediated dilation in 
patients with cardiac syndrome X: role of inflammation. Eur Rev Med Pharmacol Sci 2011; 15: 1074-7.

7. Folsom AR, Aleksic N, Catellier D, et al. C-reactive protein and incident coronary heart disease in the Atherosclerosis Risk In Communities (ARIC) study. Am Heart J 2002; 144: 233-8

8. Lim AK, Tesch GH. Inflammation in diabetic nephropathy. Mediators Inflamm 2012; 2012: 146154.

9. Jirkovska A, Fejfarova V, Hosova J, et al. Analysis of the inflammation reaction and selected indicators of immunity in patients with an infected diabetic ulcer. Cas Lek Cesk 2002; 141: 483-6.

10. Eibl N, Spatz M, Fischer GF, et al. Impaired primary immune response in type 1 diabetes: results from a controlled vaccination study. Clin Immunol 2002; 103: 249-5.

11. Gibson PH, Cuthbertson BH, Croal BL, et al. Usefulness of neutrophil/lymphocyte ratio as predictor of new-onset atrial fibrillation after coronary artery bypass grafting. Am J Cardiol 2010; 105: 186-91.

12. Tamhane UU, Aneja S, Montgomery D, et al. Association between admission neutrophil to lymphocyte ratio and outcomes in patients with acute coronary syndrome. Am J Cardiol 2008; 102: 653-7.

13. Retnakaran R, Cull CA, Thorne KI, et al.; UKPDS Study Group. Risk factors for renal dysfunction in type 2 diabetes: U.K. Prospective Diabetes Study 74. Diabetes 2006; 55: $1832-9$

14. Garg JP, Bakris GL. Microalbuminuria: marker of vascular dysfunction, risk factor for cardiovascular disease. Vasc Med 2002; 7: 35-43.

15. Remuzzi G, Bertani T. Pathophysiology of progressive nephropathies. N Engl J Med 1998; 339: 1448-56.

16. McIntyre NJ, Taal MW. How to measure proteinuria? Curr Opin Nephrol Hypertens 2008; 17: 600-3.

17. Gai M, Motta D, Giunti S, et al. Comparison between 24-h proteinuria, urinary protein/creatinine ratio and dipstick test in patients with nephropathy: patterns of proteinuria in dipstick-negative patients. Scand I Clin Lab Invest 2006; 66: 299-307.

18. Pickup J, Crook M. Is type II diabetes mellitus a disease of the innate immune system. Diabetologia 1998; 41: 1241-8.

19. Navarro JF, Mora C. Role of inflammation on diabetic complications. Nephrol Dial Transplant 2005; 20: 2601-4.

20. Rivero A, Mora C, Muros M, García J, Herrera H, Navarro-González JF. Pathogenic perspectives for the role of inflammation in diabetic nephropathy. Clin Sci 2009; 116: 479-92.

21. Spranger J, Kroke A, Mohlig M, et al. Inflammatory cytokines and the risk to develop type 2 diabetes: results of the prospective population-based European prospective investigation intra cancer and nutrition(EPIC)-postdam study. Diabetes 2003; 52: 812-7.

22. Zahorec R. Ratio of neutrophil to lymphocyte counts-rapid and simple parameter of systemic inflammation and stress in critically ill. Bratisl Lek Listy 2001; 102: 5-14.

23. Kahraman C, Yümün, G, Kahraman NK, et al. Neutrophil-to-lymphocyte ratio in diabetes mellitus patients with and without diabetic foot ulcer. Eur J Med Sci 2014; 1: 8-13.

24. Agarwal R, Light RP. Patterns and prognostic value of total and differential leukocyte count in chronic kidney disease. Clin J Am Soc Nephrol 2011; 6: 1393-9.

25. Afşar B. The relationship between neutrophil lymphocyte ratio with urinary protein and albumin excretion in newly diagnosed patients with type 2 diabetes. Am J Med Sci 2013; 347: 217-20.
26. Abdul-Rahman IS. Urine albumin/creatinine ratio: a reliable marker of renal injury in sickle cell nephropathy. Saudi J Med Med Sci 2014; 2: 17-23.

27. Watts GF, Powrie JK, O'Brien SF, Shaw KM. Apolipoprotein $\mathrm{B}$ independently predicts progression of very - low-level albuminuria in insulin-dependent diabetes mellitus. Metabolism 1996; 45: 1101-7.

28. Akbaş EM, Demirtas L, Ozcicek A, et al. Association of epicardial adipose tissue, neutrophil-to-lymphocyte ratio and platelet-to-lymphocyte ratio with diabetic nephropathy. Int J Clin Exp Med 2014; 7: 1794-801.

29. Sela S, Shurtz-Swirski R, Cohen-Mazor M, et al. The primed peripheral polymorphonuclear leukocyte a culprit underlying chronic low-grade inflammation and systemic oxidative stress in chronic kidney disease. J Am Soc Nephrol 2005; 16: 2431-8.

30. Okyay GU, Inal S, Oneç K, et al. Neutrophil to lymphocyte ratio in evaluation of inflammation in patients with chronic kidney disease. Ren Fail 2013; 35: 29-36.

31. Akbaş EM, Hamur H, Demirtas L, et al. Predictors of epicardial adipose tissue in patients with type 2 diabetes mellitus. Diabetol Metabol Syndr 2014; 6: 55.

32. Huang W, Huang J, Liu Q, et al. Neutrophil-lymphocyte ratio is a reliable predictive marker for early-stage diabetic nephropathy. Clin Endocrinol (Oxf) 2015; 82: 229-33. 